\title{
Föderale Regelungsbefugnisse für Verbandsklagerechte im Tierschutzrecht
}

\author{
Von Matthias Rossi*
}

Angesichts der bisherigen Gesetzgebung in einigen und der aktuellen

Gesetzgebungsaktivitäten in anderen Bundesländern, angesichts aber auch der Ablehnung eines tierschutzrechtlichen Verbandsklagerechts auf Bundesebene untersucht der Beitrag die Frage, wem im Bundesstaat die Gesetzgebungskompetenz für die Einführung einer

Verbandsklage im Tierschutzrecht zusteht.

\section{Einführung}

„Wer weniger Tierschutz durchsetzen will, darf klagen. Wer den Tieren zu dem Schutz verhelfen will, der ihnen rechtlich zusteht, dem sind dagegen die Hände gebunden“, so beschrieb der Präsident des Deutschen Tierschutzbundes e.V. die rechtliche Situation auf Bundesebene. ${ }^{1}$ Zur Herstellung einer „Waffengleichheit“活 setzen sich daher vor allem Tierschutzorganisationen und einige politische Parteien vehement für die Einführung einer Verbandsklage im Tierschutzrecht ein, dem Modell der Verbandsklage im Umweltrecht folgend. Da auf Bundesebene bislang alle politischen Versuche scheiterten, ein Klagerecht für Tierschutzorganisationen zu normieren, ${ }^{3}$ ergriffen einzelne Bundesländer die Initiative und schufen Verbandsklagerechte auf Landesebene. Bislang haben die Länder Bremen (2007), ${ }^{4}$ Nordrhein-Westfalen (2013), ${ }^{5}$ Saarland (2013), ${ }^{6}$ Hamburg (2013), ${ }^{7}$ Rheinland-Pfalz (2014), ${ }^{8}$

\footnotetext{
* Der Aufsatz basiert auf dem Vortrag, den der Verfasser am 24.6.2016 auf einer gemeinsam von der Forschungsplattform Recht, der Albert Schweitzer Stiftung für unsere Mitwelt, der ERNA-GRAFF-Stiftung für den Tierschutz und Röttgen, Kluge \& Hund mbB veranstalteten Tagung an der Humboldt-Universität zu Berlin gehalten hat. Eine längere Fassung des Vortrags erscheint im von Michael Kloepfer herausgegebenen Tagungsband „Die tierschutzrechtliche Verbandsklage“, Berlin 2016. Dank gilt wiss. Mitarbeiter Thomas Pardeller für die Unterstützung bei der Vorbereitung des Vortrags.

Sämtliche Internetquellen wurden zuletzt am 12.7.2016 aufgerufen.

${ }^{1}$ Deutscher Tierschutzbund e.V., Tierschutz-Verbandsklage im Bundestag, Pressemeldung v. 5.6.2013, www.tierschutzbund.de/news-storage/recht/050613-verbandsklage-bundestag.html.

${ }^{2}$ Caspar, Tierschutz im Recht der modernen Industriegesellschaft, 1999, S. 498 f.

${ }^{3}$ Die Initiative der Bundestagsfraktion Bündnis 90/Die Grünen vom 21.11.1995 (BT-Drs. 13/3036, S. 17) wurde abgelehnt (vgl. Plen.-Prot. 13/207, S. 18918). Die BR-Initiative des Bundeslandes Schleswig-Holstein vom 19.2.2004 (BR-Drs. 157/04) fand keine Mehrheit (BR-Drs. 157/04(B)). Die Initiative der Bundestagsfraktion Bündnis 90/Die Grünen vom 23.5.2012 (BT-Drs. 17/9783) wurde in zweiter Beratung am 13.12.2012 zurückgewiesen (vgl. Plen.-Prot. 17/214, S. 26368).

${ }^{4}$ Gesetz über das Verbandsklagerecht für Tierschutzvereine (TSVbeklG) v. 25.9.2007 (Brem. GB1. 2007, S. 455), zuletzt geändert durch Geschäftsverteilung des Senats v. 13.12.2011 (Brem. GB1. 2012, S. 24).

${ }^{5}$ Gesetz über das Verbandsklagerecht und Mitwirkungsrechte für Tierschutzvereine (TierschutzVMG NWR) v. 25.6.2013 (GV. NRW. 2013, S. 416).

${ }^{6}$ Gesetz Nr. 1810 über das Verbandsklagerecht für anerkannte Tierschutzverbände

(Tierschutzverbandsklagegesetz - TSVKG) v. 26.6.2013 (AB1. I 2013, S. 268).
} 
Baden-Württemberg (2015) ${ }^{9}$ und Schleswig-Holstein (2015) ${ }^{10}$ entsprechende Gesetze erlassen. Bei allen Unterschieden im Detail zielen diese Gesetze darauf ab, anerkannten Tierschutzvereinen die Möglichkeit zu geben, die im TierSchG verankerten Interessen von Tieren verwaltungsgerichtlich durchzusetzen, ohne die Verletzung eigener Rechte geltend machen zu müssen. ${ }^{11}$ Sie schaffen also sämtlich die Möglichkeit einer altruistischen Verbandsklage. ${ }^{12}$

Rechtspolitisch ist das Instrument der tierschutzrechtlichen Verbandsklage durchaus umstritten. ${ }^{13}$ Die Befürworter gehen stets von der Prämisse eines Vollzugsdefizits, jedenfalls aber eines Kontrolldefizits im Tierschutzrecht sowie von der Annahme aus, dass Tiere einen (weiteren) Stellvertreter benötigten, um ihre Interessen (gerichtlich) durchsetzen zu können. Die Verbandsklage ermögliche es Tierschützern, als „Anwälte“ der Tiere aufzutreten. Die Gegner bzw. die Skeptiker einer tierschutzrechtlichen Verbandsklage zweifeln demgegenüber schon an diesen Prämissen. Die verschiedenen Kontrollinstrumente im Tierschutzrecht seien ausreichend, um ein Vollzugsdefizit zu verhindern bzw. zu offenbaren und zu beseitigen. Zudem fungierten namentlich die Veterinärmediziner bereits nach geltendem Recht als fachkundige Anwälte der Tiere. Darüber hinaus wird befürchtet, die Verbandsklage führe zu einer Prozessflut und somit zu einer Überlastung der Gerichte, zudem würden die verwaltungsverfahrensrechtlichen Verfahren in die Länge gezogen. Schließlich sei das Strafrecht ein geeignetes Mittel, um tierschutzrechtswidrige Zustände zu ahnden.

Entgegen diesem politischen Streit über die Einführung einer tierschutzrechtlichen Verbandsklage fällt die Praxis recht nüchtern aus - von der tierschutzrechtlichen Verbandsklage ist in den Ländern, in denen sie möglich ist, bislang kaum Gebrauch gemacht worden. ${ }^{14}$ Nun darf die praktische Bedeutung der tierschutzrechtlichen Verbandsklage nicht allein von der Zahl und dem Ausgang gerichtlicher Verfahren beurteilt werden. Vielmehr kann schon die präventive Wirkung möglicher Verbandsklagen zu einem verbesserten

\footnotetext{
${ }^{7}$ Hamburgisches Gesetz über das Verbandsklagerecht für Tierschutzvereine (Hamburgisches

Tierschutzverbandsklagegesetz - HmbTierSchVKG) v. 21.5.2013 (HmbGVB1. 2013, S. 248).

${ }^{8}$ Gesetz über Mitwirkungsrechte und das Verbandsklagerecht für anerkannte Tierschutzvereine (TierSchLMVG)

v. 3.4.2014 (GVBl. 2014, S. 44), zuletzt geändert durch Gesetz v. 27.11.2015 (GVB1. 2015, S. 383).

${ }^{9}$ Gesetz über Mitwirkungsrechte und das Verbandsklagerecht für anerkannte Tierschutzorganisationen (TierSchMVG) v. 12.5.2015 (GBl. 2015, S. 317).

${ }^{10}$ Gesetz zum Tierschutz-Verbandsklagerecht (TierSchVKG SH) v. 22.1.2015 (GVOB1. 2015, S. 44).

${ }^{11}$ Rheinland-Pfalz LT-Drs. 16/2712, S. 8; vgl. § 1 TSVbeklG, § 1 I HmbTierSchVKG.

${ }^{12} \mathrm{Zu}$ den Arten und Begrifflichkeiten von Verbandsklagen vgl. z.B. Schmidt/Schrader/Zschiesche, Die Verbandsklage im Umwelt- und Naturschutzrecht, 2014, S. 1 f.; differenziert auch Schlacke, in: Schlacke/Schrader/Bunge, Aarhus-Handbuch, 2010, S. 393 ff.

${ }^{13}$ Ein Überblick über den Meinungsstand findet sich bspw. bei Raspé, Die tierliche Person, 2012, S. 324 ff.

${ }^{14} \mathrm{Zu}$ empirischen Untersuchungen vgl. Schmidt/Zschiesche/Tryjanowski, NuR 2012, 77 ff.
} 
Vollzug des materiellen Tierschutzrechts führen. Denn letztlich entfaltet jedes

Kontrollinstrument mittelbare Kontrollwirkungen schon durch den möglichen, nicht erst durch seinen tatsächlichen Gebrauch. ${ }^{15}$ Vor diesem Hintergrund ist es verständlich, dass Forderungen nach einer möglichst flächendeckenden bundesweiten Verbandsklagemöglichkeit für Tierschutzvereine weiter aufrechterhalten werden.

\section{Pflicht zur Einführung einer Verbandsklage?}

Die Einführung einer tierschutzrechtlichen Verbandsklage wird in der politischen Diskussion zuweilen als verfassungsrechtliche Pflicht dargestellt.

\subsection{Keine Verpflichtung aus Art. 20a GG}

Zur Begründung einer solchen verfassungsrechtlichen Pflicht wird überwiegend die Staatszielbestimmung des Art. 20a GG bemüht. Seit 2002 schützt der Staat nicht nur „die natürlichen Lebensgrundlagen“, sondern auch „die Tiere“. Doch so verständlich es auch sein mag, die politische Forderung nach einer tierschutzrechtlichen Verbandsklage verfassungsrechtlich zu unterfüttern, so wenig kann dies Argument bei rechtlicher Betrachtung durchdringen. Generell darf die Verfassung in einer Demokratie - auch in einer rechtsstaatlichen - nicht als deziosonistisches Programm begriffen werden. Mit ihrer limitierenden Funktion zieht die Verfassung den staatlichen Gewalten äußere Grenzen, innerhalb derer sich „der politische Wille“ durch Argument und Gegenargument formt und letztlich in einem geregelten Verfahren durch Mehrheitsbeschluss eine rechtliche und damit verbindliche Gestalt annimmt. Wer das Verfassungsrecht vorschnell als Argument bemüht, verkennt die Gestaltungsfähigkeit und -bedürftigkeit sämtlicher Lebensbereiche, ignoriert die demokratische Entscheidungsfindung und bringt auch fehlenden Respekt gegenüber förmlich getroffenen Mehrheitsentscheidungen zum Ausdruck. Art. 20a GG ist gegenüber diesem demokratischen Entscheidungsverfahren aber ganz bewusst offen, wie der - mit Blick auf Art. 20 Abs. 3 GG redundante - Passus ,im Rahmen der verfassungsmäßigen Ordnung durch die Gesetzgebung “ zeigt. ${ }^{16}$ Selbst wenn man im Übrigen der pathozentrischen Auslegung der verfassungsrechtlichen Tierschutzbestimmung folgte, wäre noch nicht entschieden, wer „Anwalt" der Tiere oder Treuhänder ihre Interessen sein sollte. ${ }^{17}$ Neben den zahlreichen

\footnotetext{
${ }^{15} \mathrm{Vgl}$. zu den Kontrollzielen allgemeiner Informationsfreiheitsgesetze Rossi, Informationszugangsfreiheit und Verfassungsrecht, 2004, S. 94 f., S. 235 ff.

${ }^{16}$ Die Gestaltungsfreiheit des Gesetzgebers - bis zu einer durch das Untermaßverbot markierten Grenze betonend Faller, Staatsziel ,Tierschutz“, 2004, S. 200 ff.

${ }^{17}$ Nicht diese Frage beantwortend, aber gleichwohl lesenswert Brandt, Merkur 7/2016, 5 ff.
} 
Tierschutzverbänden kämen insofern auch die Veterinärärzte, kämen Kommission, kämen etwa auch neu zu schaffende Ämter in Betracht. Hier offenbart sich die Gestaltungsfreiheit des Gesetzgebers, der zwar dazu angehalten ist, die notwendigen Maßnahmen zum Schutz der Tiere zu treffen, ${ }^{18}$ dabei aber über einen erheblichen Beurteilungs- und Entscheidungsspielraum verfügt. ${ }^{19}$ Er muss in Abwägung mit anderen verfassungsrechtlichen Belangen und im vorgesehenen verfassungsrechtlichen Verfahren entscheiden, welches Schutzniveau er wählen möchte und welche konkreten Instrumente er zur Verbesserung des Tierschutzes erlassen will, mithin wer Sachwalter der Tiere und deren Interessen sein soll. Art. 20a GG ist mit Blick auf die Verbandsklage insofern neutral: Weder verpflichtet die Staatszielbestimmung den bzw. die Gesetzgeber, die Verbandsklage im Tierschutzrecht einzuführen, ${ }^{20}$ noch schließt die Norm diese Möglichkeit aus. ${ }^{21}$

\subsection{Gleichlauf zu Klagemöglichkeiten ,zu Lasten“ des Tierschutzes}

Auch die Begründung, eine tierschutzrechtliche Verbandsklage müsse eingeführt werden, um einen Gleichlauf mit Klagemöglichkeiten „zu Lasten“ des Tierschutzes herzustellen, ist allein ein politisches, kein verfassungsrechtliches Argument. Soweit hier eine „Ungleichbehandlung“ in Bezug auf Rechtsschutzmöglichkeiten als Grund für eine Handlungspflicht des Gesetzgebers suggeriert wird, liegt jedenfalls keine Verletzung, ja noch nicht einmal eine Beeinträchtigung des allgemeinen Gleichheitsgrundrechts vor. Art. 3 Abs. 1 GG postuliert allein eine Gleichheit ,aller Menschen“ vor dem Gesetz, nicht hingegen eine Gleichheit von Tieren und Menschen. Soweit als Vergleichsgruppe deshalb nicht unmittelbar die Tiere, sondern mittelbar die Tierschutzverbände herangezogen werden, liegt ein Zirkelschluss in dem Sinne vor, dass als Voraussetzung der Ungleichbehandlung angenommen wird, was als deren Folge erst postuliert wird. Zudem wird übersehen, dass die Klagemöglichkeiten ,zu Lasten des Tierschutzes“ allein daraus resultieren, dass sich der Tierschutz als öffentlicher Belang zur Beschränkung grundrechtlicher Freiheiten darstellt und allein die darin liegende Möglichkeit der Rechtsverletzung nach Art. 19 Abs. 4 GG einen effektiven Rechtsschutz fordert.

\footnotetext{
${ }^{18}$ Scholz, in: Maunz/Dürig (Begr.), GG Kommentar, Stand: 12/2015, Art. 20a Rdnr. 76; Murswiek, in: Sachs (Hrsg.), GG Kommentar, 7. Aufl. 2014, Art. 20a Rdnr. 51a.

${ }^{19}$ In Bezug auf das Sozialstaatsprinzip BVerfGE 100, 271, 284.

${ }^{20}$ Gärditz, in: Landmann/Rohmer (Hrsg.), Umweltrecht Kommentar, Bd. I, Stand: 1.8.2015, Art. 20a Rdnr. 63; Schulze-Fielitz, in: Dreier (Hrsg.), GG Kommentar, Bd. II, 2. Aufl. 2006, Art. 20a Rdnr. 56; Happ, in: Eyermann (Hrsg.), VwGO Kommentar, 14. Aufl. 2014, § 42 Rdnr. 173.

${ }^{21}$ So Fest/Köpernik, DVBl. 2012, 1473, 1475.
} 


\subsection{Verbandsklagerechten in anderen Rechtsbereichen}

Soweit der Gesetzgeber in anderen Rechtsbereichen, namentlich im Verbraucherschutzrecht ${ }^{22}$ und im Umweltrecht, ${ }^{23}$ Verbandsklagemöglichkeiten geschaffen hat, zwingt ihn dies nicht, sie auch im Tierschutzrecht einzuführen. Das allgemeine Gleichheitsrecht ist hier erneut nicht berührt und kann insoweit keine verfassungsrechtliche Pflicht des (zuständigen) Gesetzgebers zur Einführung von Verbandsklagen im Tierschutzrecht begründen.

\subsection{Europa- bzw. völkerrechtliche Determinierung}

Anders als im Umweltrecht, in dem mit der Aarhus-Konvention eine völkerrechtliche Verpflichtung zur Einführung einer Verbandsklage in bestimmten Bereichen besteht, ${ }^{24}$ die ihre besondere Verbindlichkeit vor allem durch eine die Konvention umsetzende EURichtlinie ${ }^{25}$ erhalten hat, ${ }^{26}$ gibt es im Tierschutzrecht keine entsprechende völker- und/oder europarechtliche Verpflichtung. Vorbehaltlich der Berufung auf die binnenmarktbezogene allgemeine Harmonisierungskompetenz des Art. 114 AEUV und abgesehen von der tierschutzrechtlichen Querschnittsklausel des Art. 13 AEUV fehlt der EU insoweit auch die Rechtsetzungskompetenz. ${ }^{27}$

\subsection{Freie politische Entscheidung}

Die Frage, ob und unter welchen Voraussetzungen und mit welchen Konsequenzen eine tierschutzrechtliche Verbandsklage einzuführen ist, liegt im Ergebnis in der freien politischen Entscheidung des zuständigen Gesetzgebers. Er kann, er muss aber nicht Tierschutzverbänden die Möglichkeit einräumen, die Einhaltung des „nur“ objektiven Tierschutzrechts durch Gerichte überprüfen zu lassen.

\section{Grundsätze der vertikalen Kompetenzverteilung}

Wenn die Einführung einer tierschutzrechtlichen Verbandsklage also im freien politischen Ermessen des Gesetzgebers liegt, ist in der föderalistischen Ordnung zu bestimmen, ob

\footnotetext{
${ }^{22} \mathrm{Vgl}$. Unterlassungsklagengesetz (UKlaG); seit der letzten Änderung durch Art. 3 des Gesetzes vom 11.4.2016, BGB1. I S. 720, sind die Abmahnbefugnisse von Verbraucherschutzverbänden auch auf datenschutzrechtliche Verstöße ausgeweitet.

${ }^{23} \mathrm{Vgl}$. 64 BNatSchG sowie $\S 2$ UmwRG.

${ }^{24} \mathrm{Zu}$ den Folgen der Aarhus-Konvention auf die Ausweitung der Klagebefugnis im Umweltrecht vgl. bspw. Schlacke/Schrader/Bunge (Fn. 12), S. 393 ff; Bruckert, NuR 2015, 541 ff.; Ekardt, NVwZ 2015, 772 ff. sowie die Beiträge von Franzius und Krüper, in: Kloepfer (Hrsg.), Rechtsschutz im Umweltrecht, 2014, S. 145 ff. und $163 \mathrm{ff}$.

${ }^{25}$ Vgl. Art. 4 RL 2003/35/EG vom 26.5.2003.

${ }^{26} \mathrm{Zu}$ dem Charakter eines europäisiertes internationalen Umweltverwaltungsrechts vgl. Rossi, in:

Möllers/Voßkuhle/Walter, Internationales Verwaltungsrecht, 2007, S. 165 ff.

${ }^{27}$ So (zur Rechtslage vor dem Vertrag von Lissabon) auch Faller (Fn. 16), S. 39 ff.
} 
insofern die politische Entscheidung des Bundes oder die der einzelnen Länder maßgeblich ist. Dies bestimmt sich ausschließlich nach der verfassungsrechtlichen Kompetenzverteilung, die ebenso abschließend wie verbindlich ist und insbesondere nicht zur Disposition, auch nicht zur konsentierten, von Bund und Ländern steht.

\subsection{Grundsatz-Ausnahme-Verhältnis zu Gunsten der Länder}

Die grundgesetzliche Kompetenzverteilung geht in Bezug auf die Gesetzgebung von einem Grundsatz-Ausnahme-Verhältnis zu Gunsten der Länder aus. Nach Art. 70 Abs. 1 GG haben die Länder im Grundsatz - und das heißt auch im Zweifel - das Recht der Gesetzgebung, soweit das Grundgesetz nicht dem Bunde Gesetzgebungsbefugnisse verleiht.

\subsection{Zugriffsrecht des Bundes im Rahmen der konkurrierenden Gesetzgebung}

Im Bereich der sog. konkurrierenden Gesetzgebung gilt dieser Grundsatz freilich nur, solange und soweit der Bund nicht von seiner Gesetzgebungskompetenz gem. Art. 72 Abs. 1 GG Gebrauch gemacht hat. In dem Maße, in dem der Bund von seiner Gesetzgebungskompetenz Gebrauch macht, tritt eine Sperrwirkung ein, die eine eigenständige gesetzliche Reglung durch die Länder ausschließt und unmittelbar zur Nichtigkeit bereits erlassener Gesetze führt. Insofern ist hervorzuheben, dass die Regelung einer tierschutzrechtlichen Verbandsklage unabhängig von der Frage, ob eine solche Verbandsklage primär prozessrechtlich oder eher materiellrechtlich verstanden wird, in die Gesetzgebungsbefugnis des Bundes fällt. Denn er darf sowohl nach Art. 74 Abs. 1 Nr. 1 GG das ,gerichtliche Verfahrens“ ausgestalten als auch nach Art. 74 Abs. 1 Nr. GG den „Tierschutz“ regeln.

\subsection{Mögliche Sperrwirkung}

Deshalb ist es durchaus denkbar, dass das bereits erlassene Bundesrecht Sperrwirkung gegenüber den Landesgesetzgebern erzeugt. Allerdings ist mit Blick auf die inhaltlichumfängliche Begrenzung der Länder durch Art. 72 Abs. 1 GG (,soweit“) von einer Sperrwirkung nur auszugehen, wenn der Bund eine „erschöpfende und damit abschließende“ Reglung getroffen hat. ${ }^{28}$ Der bloße Umstand, der Bund eine Materie überhaupt gesetzlich geregelt hat, reicht für die Annahme einer „erschöpfenden“ Regelung insofern nicht aus. Vielmehr im Rahmen einer Gesamtwürdigung des betreffenden Normkomplexes festgestellt

\footnotetext{
${ }^{28}$ Degenhart, in: Sachs (Fn. 18), Art. 72 Rdnr. 27.
} 
werden, ob eine Regelung erschöpfend ist. ${ }^{29}$ Ausschlaggebend ist, ob ein spezieller Sachverhalt umfassend und lückenlos geregelt ist oder zumindest nach dem (objektiven) Willen des Gesetzgebers umfassend und lückenlos geregelt sein sollte. ${ }^{30}$ Mit Blick auf die tierschutzrechtliche Verbandsklage kann sich eine solche Sperrwirkung zum einen aus der bundesrechtlichen Normierung der Verwaltungsgerichtsordnung (III.), zum anderen aber auch aus einer Gesamtwürdigung des TierSchG (IV.) ergeben.

\section{4. Öffnungsklausel in der VwGO}

Das gerichtliche Verfahren vor den Verwaltungsgerichten ist Gegenstand der Verwaltungsgerichtsordnung (VwGO). Mit der VwGO hat der Bundesgesetzgeber das verwaltungsgerichtliche Verfahren nach ständiger Rechtsprechung des BVerfG erschöpfend und abschließend geregelt. ${ }^{31}$ Die vollständige und umfassende Regelung sollte die Zersplitterung des Verwaltungsprozessrechts beseitigen ${ }^{32}$ bzw. soll einer solchen vorbeugen. Den Landesgesetzgebern ist es deshalb grundsätzlich untersagt, eigene Regelungen zum Verwaltungsprozessrecht treffen.

Dies gilt jedoch nicht, soweit im Bundesgesetz selbst ein Reglungsvorbehalt zugunsten der Länder enthalten ist. Insofern gewinnt $§ 42$ Abs. 2 VwGO an Bedeutung, der zwar an der Systementscheidung des Art. 19 Abs. 4 GG für einen Individualrechtsschutz festhält, indem grundsätzlich die Verletzung eines subjektiven Rechts geltend gemacht werden muss, aber nur, „,soweit gesetzlich nichts anderes bestimmt ist.“ Dieser Vorbehalt räumt den Ländern die Möglichkeit ein, die Klagebefugnis abweichend von $§ 42$ Abs. 2 VwGO zu regeln. ${ }^{33}$ Insbesondere können die Länder auf das Erfordernis der Verletzung eigener, subjektiver Rechte verzichten und landesrechtliche Klagemöglichkeiten für Verbände einzuführen. ${ }^{34}$ Allerdings ist die Kompetenz der Länder auf die jeweilige Landesverwaltung beschränkt, erstreckt sich also nicht auf die Zulassung einer Verbandsklage gegenüber Maßnahmen

\footnotetext{
${ }^{29}$ BVerfGE 7, 342, 347; Uhle, in: Maunz/Dürig (Fn. 18), Art. 72 Rdnr. 83; Degenhart, in: Sachs (Fn. 18), Art. 72 Rdnr. 27.

${ }^{30}$ BVerfGE 109, 190, 230.

${ }^{31}$ BVerfGE 20, 238, 248; 21, 106, 115; 35, 65, 73; 37, 191, 198; 83, 24, 30.

${ }^{32}$ BVerfGE 20, 238, 248; Schmidt-Aßmann/Schenk, in: Schoch/Schneider/Bier (Hrsg.), VwGO Kommentar, Bd. I, 2015, Einl. Rdnr. 66; Sodan, in: Sodan/Ziekow (Hrsg.), VwGO Großkommentar, 4. Aufl. 2014, § 42 Rdnr. 11; Stettner, in: Dreier (Fn. 20), Art. 74 Rdnr. 26.

${ }^{33}$ BVerfGE 20, 238, 249; Schmidt-Kötters, in: Posser-Wolff (Hrsg.), VwGO Kommentar, 2. Aufl. $2014, \S 42$ Rdnr. 213.

${ }^{34}$ Sodan, in: Sodan/Ziekow (Fn. 32), § 42 Rdnr. 402; Oeter, in: v. Mangoldt/Klein/Starck (Hrsg.), GG Kommentar, Bd. II, 6. Aufl. 2010, Art. 74 Rdnr. 27. Gerade mit Blick auf diese Möglichkeit schlägt Gärditz vor, die Öffnungsklausel in $\S 42$ Abs. 2 VwGO zu streichen: Gärditz, Funktionswandel der Verwaltungsgerichtbarkeit unter dem Einfluss des Unionsrechts, Gutachten D zum 71. Deutschen Juristentag 2016, Manuskript S. 38.
} 
anderer Länder oder gar des Bundes. ${ }^{35}$ Weil das TierSchG aber nach Maßgabe des Art. 84 GG von den Ländern als eigene Angelegenheit ausgeführt wird, ist die Ausklammerung des Bundesvollzugs ohne große praktische Bedeutung.

\section{Sperrwirkung des Tierschutzgesetzes?}

Die Öffnungsklausel in $§ 42$ Abs. 2 VwGO kann eine Sperrwirkung des Bundesrechts gegenüber den Landesgesetzgebern indes nicht vollständig ausschließen. Denn wenn die Verbandsklage nicht prozedural als Sonderregelung gegenüber dem Zulässigkeitserfordernis einer Klagebefugnis, sondern materiell als Instrument zur Durchsetzung des Tierschutzrechts zu verstehen ist, kann sich eine Sperrwirkung auch aus dem TierSchG ergeben.

\subsection{Tierschutzgesetz als relevanter Maßstab}

Entgegen der Behauptung, die Erweiterung der Klagemöglichkeit sei eine rein prozessuale Frage des Verwaltungsprozessrechts, die ausschließlich am Maßstab der VwGO zu messen sei, ${ }^{36}$ muss das TierSchG als Maßstab für die Beurteilung einer Sperrwirkung gegenüber landesrechtlichen Verbandsklagen herangezogen werden.

Denn die kompetenzrechtlich relevante Sachmaterie bestimmt sich nicht formal nach der Verortung in einem bestimmten Gesetz, sondern materiell nach seinem Regelungsgehalt. Die Kompetenzordnung gilt insofern nicht für ein Gesetz, sondern für jede einzelne gesetzliche Bestimmung. Der Zuordnung zu einem bestimmten Gesetz kann allein indizierende Wirkung zukommen. Insofern ist es kompetenzrechtlich irrelevant, ob die Möglichkeit einer tierschutzrechtlichen Verbandsklage etwa in der VwGO, im TierSchG, im Umweltrechtsbehelfsgesetz (UmwRG) oder in einem eigenständigen Gesetz verortet wäre. Auch in Bezug auf die umweltrechtliche Verbandsklage war umstritten, ob sie allein oder jedenfalls primär prozessual oder auch materiell geprägt war. Das UmwRG wie alle seine bisherigen Änderungen ${ }^{37}$ rekurrieren allein auf Art. 74 Abs. 1 Nr. 1 GG, ${ }^{38}$ stellen also auf den prozessualen Charakter der Verbandsklage ab. Hingegen wird hinsichtlich der Frage, ob und in welchem Ausmaß die EU Verbandsklagerechte im Umweltrecht einführen kann, auf die

\footnotetext{
${ }^{35}$ Happ, in: Eyermann (Fn. 20), § 42 Rdnr. 171.

${ }^{36}$ Kluge, Schriftliche Stellungnahme zur öffentlichen Anhörung des nordrhein-westfälischen Landtags zum Gesetzesentwurf „Verbandsklagerecht und Mitwirkungsrechte für Tierschutzvereine“ am 31.11.11, S. 11.

${ }^{37}$ Vgl. z.B. die Begründung zur Änderung des Umwelt-Rechtsbehelfsgesetzes zur Umsetzung der AltripEntscheidung des EuGH, BR-Drs. 361/15, S. 4.

${ }^{38}$ Vgl. die Begründung des Gesetzentwurfs BR-Drs. 522/06, S. 11.
} 
materielle Kompetenz im Umweltrecht verwiesen. ${ }^{39}$ Auch die Richtlinie 2003/35/EG, die die zweite und dritte Säule der Aarhus-Konvention auf eine europarechtliche Ebene hebt, ${ }^{40}$ wurde allein auf Art. 175 EGV, den Vorläufer des heutigen Art. 192 AEUV, mithin auf die umweltrechtliche Kompetenz der EU gestützt. Dieser Widerspruch lässt sich allein politisch erklären, nicht hingegen juristisch auflösen. Ganz offensichtlich wird die Argumentation ergebnisbezogen geführt. Bei rechtlicher Betrachtung kommt man - insbesondere bei zweifelhaften Fällen - dagegen nicht umhin, zur Bestimmung der maßgeblichen Kompetenzgrundlage auf den Schwerpunkt der Regelung und den überwiegenden Sachzusammenhang abzustellen.41 Auch die Zielsetzung der Regelung ist maßgeblich für die Kompetenzbestimmung.

Hervorzuheben ist insofern, dass die Verbandsklage als ein Instrument zur Durchsetzung der tierschutzrechtlichen Bestimmungen und zur Stärkung des Tierschutzes verstanden wird..$^{42} \mathrm{Sie}$ wird zuweilen gar als eine ,staatliche Förderungsmaßnahme zu Gunsten des Tierschutzes“ qualifiziert. ${ }^{43}$ Für die Zuordnung zum Kompetenztitel Tierschutz, jedenfalls aber dessen kumulative Einschlägigkeit, spricht darüber hinaus, dass die bisher erlassenen Landesgesetze nicht schlicht eine Ausnahme von dem Erfordernis einer subjektiven Rechtsverletzung im Sinne des $\S 42$ Abs. 2 VwGO machen, sondern zudem etwa auch Mitwirkungs- und vor allem auch Informationsrechte der tierschutzrechtlichen Verbände im vorgelagerten Verwaltungsverfahren normieren. Dies mag man bei sehr weiter Auslegung noch unter die Rahmenbedingungen einer verwaltungsprozessualen Regelung subsumieren, doch sehr viel näher liegt es, hier die tierschutzrechtliche Relevanz in den Vordergrund zu stellen.

\subsection{Abschließende Regelung?}

Auch das TierSchG hindert die Länder nur dann an der Einführung einer tierschutzrechtlichen Verbandsklage, wenn es als erschöpfende und abschließende Regelung zu qualifizieren ist. Erkennbar ist die Verbandsklage im TierSchG jedoch weder ausdrücklich positiv normiert noch explizit ausgeschlossen. Von einer abschließenden Regelung kann deshalb nur ausgegangen werden, wenn der Bundesgesetzgeber die Verbandsklage bewusst nicht geregelt

\footnotetext{
${ }^{39}$ Vgl. z.B. Epiney, NVwZ 1999, 485, $491 \mathrm{f}$.

${ }^{40} \mathrm{Zu}$ dieser Form des europäisierten internationalen Verwaltungsrechts vgl. die Beiträge von Durner und Rossi, in: Möllers/Voßkuhle/Walter (Fn. 26), S. 121 ff. und 165 ff.

${ }^{41}$ Degenhart, in: Sachs (Fn. 18), Art. 70 Rdnr. 62.

${ }^{42}$ Vgl. z.B. die Gesetzesbegründung im Saarland, Saarland LT-Drs. 15/385, 8; ebenso Stellungnahme der DJGT zum Entwurf eines Gesetzes über das Verbandsklagerecht und die Mitwirkungsrechte von Tierschutzvereinen in Sachsen-Anhalt v. 7.9.14, S. 3; ähnlich offen selbst Kluge (Fn. 36), S. 4 f.

${ }^{43}$ Vgl. Caspar, DÖV 2008, 145, 148.
} 
hat. ${ }^{44}$ Insofern muss eine Gesamtwürdigung des gesamten TierSchG vorgenommen werden, muss auf das Gesetz selbst, den Regelungszweck, die Entstehungsgeschichte und die Gesetzesmaterialien zurückgegriffen werden. ${ }^{45}$

\subsubsection{Indizwirkung der Zulässigkeit von Verbandsklagen im Naturschutzrecht?}

Diese erforderliche Gesamtwürdigung wird nicht durch eine Entscheidung des BVerwG überflüssig, ${ }^{46}$ mit der das Gericht die Zulässigkeit einer landesrechtlich eingeräumten Verbandsklagerecht im überwiegend bundesrechtlich geregelten Naturschutzrecht im Ergebnis bejaht, eine Sperrwirkung des BNatSchG mithin verneint hat. ${ }^{47}$ Denn dieses Urteil kann nicht auf die Zulässigkeit einer landesrechtlichen Verbandsklage im Tierschutzrecht übertragen werden, weil das damalige BNatSchG aus dem Jahre 1977 als Rahmengesetz i.S.d. Art. 75 Abs. 1 GG a.F. erlassen wurde und dementsprechend von vorneherein auf Konkretisierung und Ausfüllung durch die Länder zugeschnitten war. Dem Bund war die umfassende und erschöpfende Regelung der Sachmaterie - anders als im Bereich der konkurrierenden Gesetzgebung - gerade verboten. ${ }^{48}$

\subsubsection{Unzulässiger Schluss von der Kompetenz auf ihren Gebrauch}

Mit der Entstehungsgeschichte des TierSchG ist daran zu erinnern, dass für bundesweite Tierschutzregelungen zunächst 1971 im Wege der Verfassungsänderung eine entsprechende Kompetenz des Bundes geschaffen werden musste, von dem der Bund dann im Jahre 1972 durch den Erlass des TierSchG Gebrauch gemacht hat. Insofern liegt die Annahme nahe, dass der zeitlich-sachliche Zusammenhang zwischen der verfassungsrechtlichen Begründung des TierSchG und ihrem Gebrauch durch den Gesetzgeber für einen abschließenden Charakter des TierSch spricht. ${ }^{49}$ Denn ausweislich der Gesetzesmaterialien sollte die rechtliche Zersplitterung durch ein „neuzeitliches, bundeseinheitliches Tierschutzrecht“ ersetzt werden. ${ }^{50}$ Tatsächlich ist der Erlass des TierSchG eng mit der Grundgesetznovelle verbunden ist. Der Bundesgesetzgeber wollte bereits in der 4. und 5. Legislaturperiode ein

\footnotetext{
${ }^{44} \mathrm{Zu}$ den Voraussetzungen eines absichtsvollen Unterlassens vgl. bspw. Uhle, in: Maunz/Dürig (Fn. 18), Art. 72 Rdnr. 93.

${ }^{45}$ BVerfGE 98, 265, 300.

${ }^{46}$ So z.B. Caspar (Fn. 43), 150.

${ }^{47}$ BVerwG, Urt. v. 29.4.1993 - 7 A 3/92, NVwZ 1993, 891.

${ }^{48}$ Rozek, in: Mangoldt/Klein/Starck (Fn. 34), Art. 75 Rdnr. 16 ff.

${ }^{49}$ Löwer, WissR, Beiheft 16, S. 114, 124.

${ }^{50} \mathrm{Vgl}$. die entsprechende Gesetzesbegründung, BT-Drs. 6/101, S. 3.
} 
Bundestierschutzgesetz erlassen ${ }^{51}$, war hierzu allerdings kompetenzrechtlich nicht befugt. So waren die gescheiterten Gesetzesinitiativen der Auslöser für die Neufassung des Art. 74 Abs. 1 GG, die dem Bund erst die umfassende Kompetenz zur Schaffung einer „bundeseinheitlichen Lösung“ eröffnete..$^{52}$ Der Gesetzgeber war bei Erlass des TierSchG deshalb auch selbst der Auffassung, die neuen Herausforderungen im Spannungsfeld zwischen technischem Fortschritt und Tierschutz „nur im Rahmen eines neuzeitlichen, bundeseinheitlichen TierSchG“ lösen zu können. ${ }^{53}$ Viel spricht insofern für eine erschöpfende Regelung dies Tierschutzrechts. ${ }^{54}$

Dem Regelungswillen des Bundesgesetzgebers kann allerdings nur indizielle Bedeutung zukommen, ${ }^{55}$ schon weil anderenfalls jede tatsächliche Fortentwicklung des Regelungsbereichs ignoriert werden müsste. Im Übrigen darf nicht zwingend von einer umfassenden Kompetenzzuweisung auf den Umfang ihres Gebrauchs geschlossen werden. ${ }^{56}$ Vielmehr muss auch insofern die Entwicklungsoffenheit des jeweiligen Sachgebiets mitbedacht werden. Deutlicher: Was 1972 als umfassende Regelung des Tierschutzrechts verstanden wurde oder jedenfalls verstanden werden konnte, kann sich in Ansehung neuer wissenschaftlicher Erkenntnisse und gewandelter politischer Einschätzungen im Jahre 2016 unter Umständen nur als Teilbereich darstellen. Die Kompetenzzuweisung in Art. 74 Abs. 1 Nr. 20 GG ist insofern offen - sie bezieht sich stets auf den aktuellen Erkenntnisstand. Das TierSchG selbst hinkt solchen Entwicklungen dagegen hinterher - es muss regelmäßig angepasst werden und kann nicht auf Dauer beanspruchen, den Regelungsbereich des Tierschutzes stets abschließend zu regeln.

\subsubsection{Erschöpfende Regelung durch Novellen von 1986 und 1992?}

Allerdings hat der Bundesgesetzgeber durch zahlreiche Änderungen des TierSchG weiter von seiner Kompetenz aus Art. 74 Abs. 1 Nr. 20 GG Gebrauch gemacht. Je konkreter und detaillierter ein Sachbereich aber normiert ist, desto eher ist davonauszugehen, dass der Gesetzgeber eine erschöpfenden Regelung i.S.d. Art. 72 Abs. 1 GG treffen wollte. ${ }^{57}$ Zur

\footnotetext{
${ }^{51}$ BT-Drs. 6/85 und BT-Drs. 5/934.

${ }^{52}$ BT-Drs. 6/1584; vgl. zur Entwicklung des Tierschutzrechts etwa Caspar (Fn. 2), S. 277 ff.

${ }^{53}$ BT-Drs. 6/2559, S. 9.

${ }^{54}$ Kunig, in: v. Münch/Kunig (Hrsg.), GG Kommentar, Bd. II, 6. Aufl. 2012, Art. 72 Rdnr. 9; Degenhart, in:

Sachs (Fn. 18), Art. 72 Rdnr. 27; Uhle, in: Maunz/Dürig (Fn. 18), Art. 72 Rdnr. 88.

${ }^{55}$ Stettner, in: Dreier (Fn. 20), Art. 74 Rdnr. 26; Oeter, in: v. Mangoldt/Klein/Starck (Fn. 34), Art. 72 Rdnr. 73 ;

Kunig, in: v. Münch/Kunig (Fn. 54), Art. 72 Rdnr. 9.

${ }^{56}$ Im Ergebnis ebenso Caspar (Fn. 43), 147.

${ }^{57}$ Uhle, in: Maunz/Dürig (Fn. 18), Art. 72 Rdnr. 87.
} 
Verbandsklage im Tierschutzrecht hat sich der Gesetzgeber in diesen Änderungen zwar nicht verhalten. Er hat aber zahlreiche andere Instrumente eingeführt, die zu einer Verbesserung des Tierschutzes und zu einer Vollzugskontrolle beitragen sollen.

Das gilt zunächst für die Tierschutzbeauftragten, die nach $\S 10$ TierSchG allen Einrichtungen, die Tierversuche unternehmen, verpflichtend vorgeschrieben ist. Sie haben nach $\S 5$ IV Nr. 1 TierSchVersV die Aufgabe, auf die „Einhaltung von Vorschriften, Bedingungen und Auflagen im Interesse des Tierschutzes zu achten.“ Ihnen kommt somit eine Garantenstellung für das Wohlergehen der in der Einrichtung befindlichen Tiere zu, die sie dazu verpflichtet, etwaige Verstöße gegen tierschutzrechtliche Bestimmungen zu beseitigen. ${ }^{58}$ Eine Verletzung ihrer gesetzlichen Pflichten ist zudem strafrechtlich sanktioniert. ${ }^{59}$ Die Tierschutzbeauftragten lassen sich insoweit als Sachwalter der Tiere und deren Interessen verstehen.

Als weiteres Instrument zur Verbesserung des Tierschutzes ist die Tierversuchskommission in $\S 15$ TierSchG eingeführt worden. Sie hat die zuständigen Behörden bei der Überwachung der Einhaltung der tierschutzrechtlichen Vorschriften zu unterstützen. ${ }^{60}$ In der

Tierversuchskommission sind gemäß $§ 42$ Abs. 2 TierSchVersV auch die

Tierschutzorganisationen mit mindestens einem Drittel aller Kommissionsmitglieder vertreten. ${ }^{61}$ Die Tierschutzverbände werden auf diesem Weg beteiligt und können bei Behördenentscheidungen mitwirken. Dies könnte dafür sprechen, dass der Gesetzgeber eine abschließende Reglung in Bezug auf die Beteiligung der Verbände treffen wollte.

Schließlich hat der Gesetzgeber im Jahre 1992 noch eine Regelung über Schiedsverfahren bei Tiertransporten in das TierSchG aufgenommen ( $§ 16 \mathrm{i}$ TierSchG). Hierbei handelt es sich um ein besonderes Verfahren außerhalb des verwaltungsrechtlichen Rechtsschutzes. Behörde und Transporteur können ihren etwaigen Streit außergerichtlich durch den Schiedsspruch eines Sachverständigen aus dem von der EU erstellten Verzeichnis beilegen. Selbst wenn der Gesetzgeber mit dieser Norm fremdbestimmt eine EU-Richtlinie umgesetzt hat, die ihrerseits primär die Verwirklichung des europäischen Binnenmarkts im Blick hatte, ${ }^{62}$ kann die

\footnotetext{
${ }^{58}$ So Hirt/Maisack/Moritz, TierSchG Kommentar, 3. Aufl. 2016, TierSchVersV $§ 5$ Rdnr. 10; Lorz/Metzger, TierSchG, 6. Aufl. 2008, Einf. Rdnr. 124.

${ }^{59}$ Goetschel, in: Kluge (Hrsg.), TierSchG, 2002, § 8b Rdnr. 5.

${ }^{60}$ Vgl. BT-Drs. 10/3158, S. 28; Kluge, in: Kluge (Fn. 59), § 15 Rdnr. 2.

${ }^{61}$ Hirt/Maisack/Moritz (Fn. 58), TierSchVersV § 42 Rdnr. 2.

${ }^{62}$ Hirt/Maisack/Moritz (Fn. 58), TierSchG $\S \S 16 f-16 i$ Rdnr. 1.
} 
detaillierte und spezielle Regelung doch durchaus Sperrwirkung für Verbandsklagen im Bereich des Tiertransportrechts entfalten. ${ }^{63}$

Obwohl der Bundesgesetzgeber mit den Novellierungen des TierSchG in den Jahren 1986 und 1992 erneut von seiner Kompetenz aus Art. 74 Abs. 1 Nr. 20 GG Gebrauch gemacht hat, muss bezweifelt werden, dass das TierSchG damit einen abschließenden Charakter erhalten hat, der die Länder von der Einführung einer Verbandsklage ausschließt. ${ }^{64}$ Denn die Verbandsklage ist weder explizit im Gesetzestext noch implizit im Gesetzgebungsverfahren ausgeschlossen worden. Die eingeführten Instrumente sind zudem nur beratender bzw. sektoraler Art, während eine Verbandsklage umfassend auf den Vollzug des gesamten Tierschutzrechts bezogen sein könnte. ${ }^{65} \mathrm{Im}$ Vergleich zu den drei skizzierten Instrumenten stellt sich das verwaltungsprozessrechtliche Instrumente einer Verbandsklage als aliud dar. Allein aus der Normierung anderer Instrumente zur Verbesserung des Tierschutzes lässt sich nicht auf den Ausschluss einer tierschutzrechtlichen Verbandsklage auf Landesebene schließen.

\subsubsection{Keine Ermächtigung an den Landesgesetzgeber}

Eine Sperrwirkung gegenüber den Landesgesetzgebern ergibt sich auch nicht daraus, dass das TierSchG einen Vorbehalt zu Gunsten der Landesgesetzgebung enthält, der im Umkehrschluss erkennen lassen würde, dass landesrechtliche Regelungen im Übrigen ausgeschlossen sein sollten. ${ }^{66}$ Denn das TierSchG enthält keinen solchen Vorbehalt. Zwar ermächtigt $\S 13 \mathrm{~b}$ TierSchG die Landesregierungen zum Erlass einer Rechtsverordnung in Bezug auf freilebene Katzen ermächtigt. Dieser Bestimmung kann aber nicht entnommen werden, dass das TierSchG im Übrigen abschließend wäre. Denn mit dieser Vorschrift grenzt das TierSchG nicht den Sachbereich des formellen Bundesgesetzgebers von dem der formellen Landesgesetzgeber ab, sondern delegiert nach Maßgabe und im Einklang mit Art. 80 GG einen Teilbereich des von ihm beanspruchten Regelungsbereichs an die Landesregierungen als Verordnungsgeber. Anders als die formellen Landesgesetzgeber sind die Landesregierungen dabei an Inhalt, Zweck und Ausmaß der gesetzlichen Verordnungsermächtigung gebunden.

\footnotetext{
${ }^{63}$ Vgl. Caspar (Fn. 43), 150.

${ }^{64}$ Zweifelnd ebenso v. Loeper, in: Kluge (Fn. 59), Einf. Rdnr. 169; Hirt/Maisack/Moritz (Fn. 58), Einf. Rdnr. 92; Caspar (Fn. 43), 152; a.A. Löwer (Fn. 49), S. 124.; Metzger, in: Erbs/Kohlhaas, Strafrechtliche Nebengesetze, TierSchG, Stand: $3 / 2016$, Vor $\S 1$ Rdnr. 9.

${ }^{65}$ Fest/Köpernik (Fn. 21), 1477.

${ }^{66} \mathrm{Zu}$ solchen Vermutungen vgl. BVerfGE 20, 238, 256; 21, 106, 115; 24, 367, 386.
} 


\subsubsection{Sperrwirkung durch Ablehnung einer Landesinitiative im Bundesrat?}

Zum Teil wird die Auffassung vertreten, ${ }^{67}$ der Bund hätte seinen Willen, keine tierschutzrechtliche Verbandsklage zuzulassen, dadurch zum Ausdruck gebracht, dass der Bundesrat im Jahre 2004 einen Antrag des Landes Schleswig-Holstein abgelehnt hat, der auf eine Bundesratsinitiative zur Einführung einer bundesweiten Verbandsklage im Tierschutzrecht gerichtet war. ${ }^{68}$

Allerdings repräsentiert diese Beschlussfassung im Bundesrat nicht den Willen des Bundesgesetzgebers. Denn hinsichtlich der Willensbekundung des Bundesgesetzgebers ist vorrangig auf den Bundestag zu rekurrieren, ohne dessen Beschluss nach Art. 77 Abs. 1 GG grundsätzlich ${ }^{69}$ keine Gesetze in Kraft treten können. ${ }^{70}$ Beschlüsse des Bundesrates sind für die Willensbekundung „des Bundesgesetzgebers“ zwar nicht irrelevant, wie die Möglichkeit des Einspruchs und mehr noch die Verweigerung der Zustimmung zeigen. In beiden Fällen kommt den Beschlüssen des Bundesrates maßgebliche Bedeutung für die Ermittlung des Willens „des Bundesgesetzgebers“ zu. Anders verhält es sich indes, wenn nicht über einen bereits im Bundestag verhandelten Gesetzentwurf, sondern über die Frage abgestimmt wird, ob eine Gesetzesinitiative des Bundesrates ergriffen werden soll. Der Antrag des Landes Schleswig-Holstein war keine Gesetzesinitiative, sondern nur eine Initiative zur Initiative. Mit seiner Ablehnung bekundet der Bundesrat deshalb nur seine Haltung als initiativberechtigtes Organ, nicht hingegen seine Haltung als mitwirkungsberechtigtes Gesetzgebungsorgan. Deshalb kann der Beschluss des Bundesrates, keine Initiative zur Einführung einer tierschutzrechtlichen Verbandsklage zu ergreifen, nicht als Indiz für einen absichtsvollen Regelungsverzicht des TierSchG gewertet werden.

\subsubsection{Tierschutznovelle 2013 - Ablehnung einer Gesetzesinitiative}

Anders verhält es sich indes mit der letzten Änderung des TierSchG im Jahre 2013. Denn im Zuge des 3. Änderungsgesetzes, mit dem auf Initiative der Bundesregierung ${ }^{71} \mathrm{im}$ Wesentlichen die Versuchstier-Richtlinie der EU ${ }^{72}$ umgesetzt werden sollte, brachte die

\footnotetext{
${ }^{67}$ Stellungname der DGAR zum Gesetzesentwurf des Tierschutzverbandsklagerechts v. 9.4.2013, SchleswigHolstein LT-Umdruck 18/1060, S. 5.

${ }^{68}$ BR-Drs. 157/04.

${ }^{69}$ Etwas anderes gilt nur für den Gesetzgebungsnotstand nach Art. 81 GG.

${ }^{70}$ Caspar (Fn. 43), 151.

${ }^{71}$ BT-Drs. 17/10572.

${ }^{72}$ Richtlinie 2010/63/EU des Europäischen Parlaments und des Rates der EU vom 22.9.2010 zum Schutz der für wissenschaftliche Zwecke verwendeten Tiere, ABl. EU 2010 L 276, S. 33.
} 
Fraktion Bündnis 90/Die Grünen einen weiteren Gesetzentwurf ein ${ }^{73}$ der explizit auf ein Verbandsklagerecht für anerkannte Tierschutzorganisationen zielte. ${ }^{74}$ Die Gesetzesinitiative wurde als Änderungsantrag im Rahmen der Beratung und Beschlussfassung des 3. Änderungsgesetzes behandelt - und im Ergebnis von der Mehrheit des Bundestages abgelehnt. ${ }^{75}$

In dieser Ablehnung kommt unmissverständlich der Wille des Bundesgesetzgebers zum Ausdruck, auf Bundesebene kein Verbandsklagerecht im Tierschutzrecht einführen zu wollen. Erkennbar wird insofern ein absichtsvoller Regelungsverzicht, ${ }^{76}$ der nach der Rechtsprechung des BVerfG nicht explizit im Gesetzestext zum Ausdruck kommen muss. ${ }^{77}$ Vielmehr ist der Wille des Gesetzgebers bereits dort feststellbar, wo sich das Parlament mit einer bestimmten Frage auseinandergesetzt hat, diese aber dann nicht weiter verfolgt bzw. nicht geregelt hat. ${ }^{78}$ Dabei ist es unerheblich, zu welchem Zeitpunkt des Gesetzgebungsverfahrens sich der Bundestag mit der entsprechenden Frage befasst hat. ${ }^{79}$ Bis zu einer erneuten Befassung des Bundestages mit dem Thema einer tierschutzrechtlichen Verbandsklage ist juristisch deshalb davon auszugehen, dass eine solche Klagemöglichkeit auf Bundesebene nicht gewollt und deshalb weder im TierSchG noch in der Verwaltungsgerichtsordnung normiert ist.

\subsection{Unterschied zwischen Regelungsverzicht auf Bundesebene und Sperrwirkung auf}

\section{Landesebene}

Dieser Regelungsverzicht auf Bundesebene führt aber noch nicht automatisch zu einer Sperrwirkung auf Landesebene. Vielmehr ist jedenfalls dann zwischen dem

Regelungsverzicht auf Bundesebene und einer Sperrwirkung auf Landesebene zu differenzieren, wenn in Bezug auf die streitige Materie bereits Landesrecht erlassen worden ist. Dies hat das BVerfG in seiner Entscheidung zum Bayerischen Schwangerenhilfegesetz in Bezug auf abweichendes Landesrecht zugunsten eines Fachärztevorbehalts deutlich zum Ausdruck gebracht: ${ }^{80}$

\footnotetext{
${ }^{73}$ BT-Drs. 17/9783.

${ }^{74}$ BT-Drs. 17/9783, S. 35.

${ }^{75}$ BT- Drs. 17/11811.

${ }^{76}$ Vgl. BVerfG, Beschl. v. 9.2.1972 - 1 BvR 111/68, NJW 1972, 859; BVerfGE 32, 319, 327 f.; BVerfG Beschl.

v. 15.10.2014 - 2 BvR 920/14, NJW 2015, 44, 45; BVerfGE 113, 348, 371.

${ }^{77}$ BVerfG, Beschl. v. 6.10.2009 - 2 BvL 5/09, NVwZ 2010, 247, 248.

${ }^{78}$ BVerfGE 98, 265, 312 ff.; Uhle, in: Maunz/Dürig (Fn. 18), Art. 72 Rdnr. 93; krit. dazu: Degenhart, in: Sachs (Fn. 18), Art. 72. Rdnr. 25.

${ }^{79}$ BVerfGE 98, 265, 313; Degenhart, in: Sachs (Fn. 18), Art. 72 Rdnr. 25.

${ }^{80}$ BVerfGE 98, 265.
} 
„Eine Sperrwirkung für abweichendes Landesrecht ist dem Gesetz indessen nicht zu entnehmen. Hierzu genügte ein Regelungsverzicht nicht. Vielmehr hätte es einer ausdrücklichen Vorschrift bedurft, da dem Bundesgesetzgeber bei seiner Novellierung die uneinheitliche Rechtslage in den Ländern bekannt war. (...) Hätte sie (die bundeseinheitliche Regelung) einen Gynäkologenvorbehalt abschließend verhindern wollen, hätte dies schon um der Rechtsklarheit willen deutlich ausgesprochen werden müssen, weil dann älteres Landesrecht außer Kraft gesetzt worden wäre. "81

Die Entscheidung des BVerfG war zwar innerhalb und außerhalb des Senats zwar sehr umstritten. ${ }^{82}$ Für die Frage nach der Sperrwirkung des TierSchG gegenüber der Einführung einer tierschutzrechtlichen Verbandsklage auf Landesebene spielen diese Meinungsverschiedenheiten aber keine Rolle. Denn der zitierte Abschnitt aus der Entscheidung war nicht von dem gerichtsinternen Streit und betroffen und ist, soweit ersichtlich, auch im Schriftum nicht kritisch kommentiert worden. Vor allem aber sind es drei wesentliche Argumente, die für die Differenzierung zwischen einem Regelungsverzicht auf Bundesebene und einer Sperrwirkung gegenüber der Landesebene sprechen:

\subsubsection{Rechtsklarheit}

Das erste Argument wird bereits vom BVerfG selbst betont: Wenn es auf Landesebene bereits Gesetze gibt, deren verfassungsrechtliche Grundlage durch die vermeintliche Sperrwirkung des Bundesgesetzes in Frage gestellt wird, dann sind an einen absichtsvollen Regelungsverzicht aus Gründen der Rechtsklarheit höhere Anforderungen zu stellen. Denn eine Sperrwirkung führte in solchen Fällen nicht nur dazu, dass die Landesgesetzgeber künftig von der Gesetzgebung ausgeschlossen sind, sondern auch zur Nichtigkeit der bereits erlassenen Landesgesetze. Zweifel an der Sperrwirkung führten deshalb bei Behörden und Gerichten wie auch bei betroffenen Privaten zu erheblicher Unsicherheit, ob das formal in Kraft befindliche Landesgesetz angewendet werden kann oder ob es mangels Kompetenz nichtig ist. Um solche Zweifel auszuschließen, fordert das BVerfG ,eine ausdrückliche Vorschrift".

\footnotetext{
${ }^{81}$ BVerfGE 98, 265, 307.

${ }^{82}$ Vgl. die Sondervoten BVerfGE 98, 265, 329 ff. u. 359 ff.
} 


\subsubsection{Bundestreue}

Neben der Rechtsklarheit wird der Bundesgesetzgeber vor allem durch den Grundsatz bundesfreundlichen Verhaltens dazu verpflichtet, eine etwaig intendierte Sperrwirkung gegenüber dem Landesgesetzgeber explizit deutlich zu machen. Der Grundsatz bundesfreundlichen Verhalten - die Bundestreue - wird als ungeschriebener Verfassungsgrundsatz unmittelbar aus dem Bundesstaatsprinzip abgeleitet. ${ }^{83}$ Er verpflichtet den Bund und die Länder im jeweilig gegenseitigen Verhältnis zu wechselseitiger Rücksichtnahme ${ }^{84}$ und verdichtet sich u.a. als Kompetenzausübungsschranke: Jede Ebene muss bei der Inanspruchnahme eines Kompetenztitels auch die Kompetenzen der jeweils anderen Ebene berücksichtigen.

Heruntergebrochen auf die konkurrierende Gesetzgebung folgt aus der Bundestreue für die Länder insoweit das Gebot, nicht kategorisch auf der Vermutung der Gesetzgebungszuständigkeit nach Art. 70 Abs. 1 GG zu beharren, wenn deutlich ist, dass der Bund von einem konkurrierenden Gesetzgebungstitel umfassend Gebrauch machen wollte. Umgekehrt verpflichtet der Grundsatz der Bundestreue den Bund erstens, nicht missbräuchlich die umfassende Inanspruchnahme eines Gesetzgebungstitels zu behaupten, um die Länder an weitergehender Gesetzgebung zu hindern, und deshalb zweitens, den umfassenden Regelungsanspruch hinreichend zum Ausdruck zu bringen. Dies gilt drittens um so mehr, wenn die Länder nicht nur künftig von der Gesetzgebung ausgeschlossen werden sollen, sondern wenn eine vorherige Gesetzgebung der Länder rückwirkend außer Kraft gesetzt werden soll. Der Bundesgesetzgeber trägt insoweit auch eine Verantwortung für in Kraft befindliches Landesrecht.

\subsubsection{Zweifelsfallregelung}

Schließlich spricht auch das in Art. 70 Abs. 1 GG verankerte Grundsatz-AusnahmeVerhältnis zu Gunsten der Länder dafür, dass der Bund eine Sperrwirkung im Bereich der konkurrierenden Gesetzgebung explizit darlegen und nicht nur stillschweigend dadurch in Anspruch nehmen kann, dass er auf Bundesebene nicht regelnd tätig wird. Kann der Wille des Bundesgesetzgebers nicht einwandfrei und eindeutig festgestellt werden, so reaktualisiert sich

\footnotetext{
${ }^{83} \mathrm{Zu}$ Einzelheiten vgl. die Darstellung bei Kloepfer, Verfassungsrecht Bd. I, 2011, § 9 Rdnr. 197 ff.

${ }^{84}$ BVerfGE 12, 205, 254.
} 
die Vermutung des Art. 70 Abs. 1 GG zu Gunsten der Länder, ist also im Zweifel von einem Verbleib der Gesetzgebungskompetenz bei den Ländern auszugehen. ${ }^{85}$

\subsection{Regelungsverzicht des Bundes ohne Sperrwirkung gegenüber den Ländern}

Folgt man dieser grundsätzlichen Differenzierung zwischen dem Regelungsverzicht auf Bundesebene und einer Sperrwirkung auf Landesebene, wird deutlich, dass die in der Zurückweisung der Gesetzesinitiative der Fraktion Bündnis 90/Die Grünen zum Ausdruck kommende Ablehnung gegenüber einer tierschutzrechtlichen Verbandsklage auf Bundesebene nicht zugleich auch eine Sperrwirkung gegenüber der Einführung einer solchen Verbandsklage in den Ländern entfaltet.

Denn als der Bundestag am 13.12.2012 den Antrag zurückgewiesen hat, ${ }^{86}$ war nicht nur das Gesetz über das Verbandsklagerecht in Bremen seit mehr als fünf Jahren in Kraft, ${ }^{87}$ sondern wurden auch bereits in den Ländern Hamburg und Nordrhein-Westfalen entsprechende Gesetzentwürfe beraten. In Hamburg war der Gesetzentwurf am 13.03.2012 in die Bürgerschaft eingebracht und am 16.05.2013 angenommen worden. ${ }^{88}$ In Nordrhein-Westfalen war der entsprechende Gesetzentwurf am 04.07.2012 in den Landtag eingebracht ${ }^{89}$ und am 19.06.2013 angenommen worden. ${ }^{90}$ Im Saarland wurde das entsprechende Gesetz zwar erst am 13.03.2013 und somit zu einem Zeitpunkt auf den Weg gebracht, als der Bundestag und der Bundesrat über das 3. Gesetz zur Änderung des TierSchG bereits beraten hatten, allerdings war es bereits zuvor in der politischen Diskussion.

Im Ergebnis musste dem Bundesgesetzgeber somit klar sein, dass eine etwaige Sperrwirkung nicht nur zur Nichtigkeit des bereits in Kraft befindlichen Bremer Gesetzes geführt hätte, sondern zugleich auch die Gesetzesvorhaben in Hamburg und Nordrhein-Westfalen untergraben hätte. Dabei muss davon ausgegangen werden, dass der Bundesgesetzgeber Kenntnis von diesen landesrechtlichen Entwicklungen hatte oder jedenfalls hätte haben müssen. Insbesondere den Mitgliedern des Ausschusses für Ernährung, Landwirtschaft und Verbraucherschutz sowie dem Bundesrat bzw. einigen seiner persönlichen Mitglieder musste bekannt sein, dass es in Bremen ein solches Verbandsklagerecht gab und in anderen Ländern

\footnotetext{
${ }^{85}$ Rengeling, in: Isensee/Kirchhof (Hrsg.), HStR, Bd. VI, 3. Aufl. 2008, § 135 Rdnr. 159; Oeter, in: v. Mangoldt/Klein/Starck (Fn. 34), Art. 72 Rdnr. 75; Uhle, in: Maunz/Dürig (Fn. 18), Art. 72 Rdnr. 84; Jarass, NVwZ 1996, 1041, 1044.

${ }^{86}$ Plen.-Prot. 17/214, S. 26368.

${ }^{87}$ Gesetz über das Verbandsklagerecht für Tierschutzvereine (TSVbeklG) v. 25.9.2007 (Brem. GB1. 2007, 455).

${ }^{88}$ Plen.-Prot. 20/60, S. 4644.

${ }^{89}$ LT-Drs. 16/77.

${ }^{90}$ Plen.-Prot. 16/33, S. $2911 \mathrm{ff}$.
} 
eingeführt wird. Unter solchen Umständen genügt ein Schweigen nicht, um eine Sperrwirkung gegenüber den Ländern zu entfalten. Vielmehr ist insofern eine explizite gesetzliche Regelung oder jedenfalls doch eine klarstellende Willensbekundung des Bundesgesetzgebers fordern. ${ }^{91}$ An dieser fehlt es indes - weder hat der Bundesgesetzgeber Verbandsklagen auf Landesebene normativ im Gesetz selbst ausgeschlossen noch ist diese Frage im Verlauf des Gesetzgebungsverfahrens überhaupt diskutiert worden. Die föderale Regelungsbefugnis für tierschutzrechtliche Verbandsklagen liegt insofern derzeit bei den Ländern.

\section{Handlungsoptionen für den Bundesgesetzgeber}

Vor dem Hintergrund dieser kompetenzrechtlichen Ausgangslage bleiben dem Bund politisch im Wesentlichen drei Handlungsoptionen.

\subsection{Untätigbleiben}

Der Bund ist auch nach der Einführung von tierschutzrechtlichen Verbandsklagen in acht Bundesländern nicht verpflichtet, seinerseits tätig zu werden. Ebenso wie die Länder nicht zur Homogenität hinsichtlich der Rechte der Tierschutzverbände verpflichtet sind, ${ }^{92}$ wird auch der Bund nicht durch die heterogene Rechtslage zur vereinheitlichenden Gesetzgebung verpflichtet. Auch die Tatbestandsmerkmale der Erforderlichkeitsklausel des Art. 72 Abs. 2 GG - so diese denn wegen des materiellen Tierschutzgehalts einer Verbandsklage einschlägig ist - vermögen keine Pflicht des Bundes zu begründen, weil sie nicht als Voraussetzungen einer Gesetzgebungspflicht, sondern als Grenzen einer Rechtsetzungsbefugnis konzipiert sind.

\subsection{Normierung eines bundeseinheitlichen Verbandsklagerecht}

Sollte die heterogene Rechtslage indes als unbefriedigend empfunden werden, könnte der Bund ein bundeseinheitliches Verbandsklagerecht für Tierschutzorganisationen normieren. Dies hätte weitreichende Konsequenzen für die Bundesländer: Sie wären nicht nur in Zukunft von der Gesetzgebung ausgeschlossen, vielmehr wären auch die bereits erlassenen Gesetze ab Inkrafttreten einer entsprechenden bundesrechtlichen Regelung nichtig, unabhängig davon, ob sie inhaltlich von der bundesrechtlichen Regelung abwichen, ihr eins zu eins entsprächen oder sie auch nur ergänzten..$^{93}$

\footnotetext{
91 Vgl. BVerfGE 49, 343, 359.

${ }^{2} \mathrm{Vgl}$. BVerfGE 49, 343, 359.

${ }^{93}$ Vgl. BVerfGE 102, 99, 115; 109, 190, 230; Rengeling, in: Isensee/Kirchhof (Fn. 85), § 135 Rdnr. 159.
} 


\subsubsection{Einschlägigkeit der Erforderlichkeitsklausel}

Allerdings wird in Bezug auf die Regelungsbefugnis des Bundes die Frage bedeutsam, ob eine Verbandsklagebefugnis (eher) dem „gerichtlichen Verfahren“ im Sinne des Art. 74 Abs. 1 Nr. 1 GG oder dem „Tierschutz“ im Sinne des Art. 74 Abs. 1 Nr. 20 GG zuzuordnen ist. Denn während das ,gerichtliche Verfahren“ in die unkonditionierte konkurrierende Gesetzgebung des Bundes nach Art. 72 Abs. 1 GG fällt, darf der Bund auf dem Gebiet des Tierschutzes nur unter den Voraussetzungen der Erforderlichkeitsklausel des Art. 72 Abs. 2 GG tätig werden. Vorbehaltlich einer genauen gesetzlichen Regelung erscheint es unausweichlich, die tierschutzrechtliche Verbandsklage wegen ihrer Zielsetzungen und vor allem auch wegen der flankierenden Bestimmungen zum vorangehenden Verwaltungsverfahren als primär tierschutzrechtliche Regelung zu qualifizieren, weshalb eine bundesrechtliche Regelung am Maßstab der Erforderlichkeitsklausel gemessen werden müsste

\subsubsection{Begründung der Erforderlichkeit}

Ob eine bundesgesetzliche Regelung zum Verbandsklagegerecht dem Maßstab der Erforderlichkeitsklausel genügen würde, ist schwer zu prognostizieren. Denn das BVerfG hat in einer (erstaunlich) konsequenten Rechtsprechung die Erforderlichkeitsklausel des Art. 72 Abs. 2 GG unter Berufung auf den Willen des verfassungsändernden Gesetzgebers von 1994 stets sehr eng interpretiert, ${ }^{94}$ gleichzeitig aber nicht zu einer unüberwindbaren Hürde modifiziert. ${ }^{95}$ Entscheidend ist vor allem, dass mit dem Wortlaut des Art. 72 Abs. 2 GG und den Entscheidungen des BVerfG die drei Alternativen des Art. 72 Abs. 2 GG - die Herstellung gleichwertiger Lebensverhältnisse, die Wahrung der Rechtseinheit im gesamtstaatlichen Interesse und die Wahrung der Wirtschaftseinheit im gesamtstaatlichen Interesse - streng voneinander zu unterscheiden und dementsprechend auch eigens zu begründen sind.

Für die tierschutzrechtliche Verbandsklage scheint dabei der 2. Alternative - der Wahrung der Rechtseinheit im gesamtstaatlichen Interesse - die größte Bedeutung zuzukommen. Diese Alternative kann nach dem BVerfG aber

\footnotetext{
${ }^{94}$ Vgl. BVerfGE 106, 62 (Altenpflegegesetz); BVerfGE 110, 141 (Kampfhunde); BVerfGE 111, 10 (Ladenschlussgesetz); BVerfGE 111, 226 (Juniorprofessur); BVerfGE 112, 226 (Studiengebühren); BVerfG, Urt. v. 21.7.2015 - 1 BvF 2/13 (Betreuungsgeld), NJW 2015, 2399.

${ }^{95} \mathrm{Vgl}$. z.B. die fast schon apodiktische Feststellung, dass für den Erlass des Gentechnikgesetz „die Voraussetzungen der Erforderlichkeitsklausel vorliegen“, BVerfGE 128, 1, 34.
} 
„nicht so verstanden werden, dass die Setzung bundeseinheitlichen Rechts stets erforderlich wäre. Unterschiedliche Rechtslagen für die Bürger sind notwendige Folge des bundesstaatlichen Aufbaus. Auf allen in Art. 74 und Art. 75 GG genannten Gebieten lässt das Grundgesetz eine Rechtsvielfalt prinzipiell zu. Einheitliche Rechtsregeln können in diesen Bereichen aber erforderlich werden, wenn eine unterschiedliche rechtliche Behandlung desselben Lebenssachverhalts unter Umständen erhebliche Rechtsunsicherheiten und damit unzumutbare Behinderungen für den länderübergreifenden Rechtsverkehr erzeugen kann. Um dieser sich unmittelbar aus der Rechtslage ergebenden Bedrohung von Rechtssicherheit und Freizügigkeit im Bundesstaat entgegen zu wirken, kann der Bund eine bundesgesetzlich einheitliche Lösung wählen."“96

Ob die heterogene Rechtslage bezüglich der Möglichkeiten einer tierschutzrechtlichen Verbandsklage tatsächlich zu erheblichen Rechtsunsicherheiten führen, kann aufgrund der wenigen Erfahrungen mit diesem Instrument noch nicht beurteilt werden.

\subsection{Aktivierung der Sperrwirkung für die Länder}

Auch bei der dritten Option des Bundes, bei der Möglichkeit, eine Sperrwirkung für die Länder zu entfalten, ohne selbst auf Bundesebene eine tierschutzrechtliche Verbandsklage einzuführen, müsste die Erforderlichkeitsklausel beachtet werden. Ein solches generelles „Verbot“ von Verbandsklagen im Tierschutzrecht dürfte nicht durch ,absichtsvollen Regelungsverzicht“" ausgesprochen werden, sondern müsste explizit gesetzlich verankert werden.

\section{Zusammenfassung und Ausblick}

Föderale Regelungsbefugnisse für Verbandsklagerechte im Tierschutzrechtstehen stehen nach derzeitiger Rechtslage sowohl dem Bund als auch den Ländern zu, dem Bund allerdings nur unter Beachtung der Erforderlichkeitsklausel, den Ländern immer nur bezogen auf ihre eigene Landesverwaltung. Weder Bund noch Länder sind dabei zur Einführung einer tierschutzrechtlichen Verbandsklage verpflichtet. Macht der Bund von seiner Gesetzgebungskompetenz Gebrauch, verlieren die Länder ihre Regelungsbefugnis. Bereits erlassene Landesgesetze würden nichtig. Dieser kompetenzrechtliche Befund zeugt nicht von einer Schwäche rechtsstaatlich gebotener Klarheit, sondern ist Ausdruck der innovativen Kraft des Föderalismus.

\footnotetext{
${ }^{96}$ BVerfGE 106, 62, 146.
} 